\title{
Fungal Detoxification of Certain Fusarium Moniliforme Mycotoxins Using Yarrowia Lipolytica with Special Emphasis on its Possible Mechanism of Action
}

\author{
Elaasser, Mahmoud Mohamed; Magdi, Hani Mohamed; Mourad, \\ Mohamed Hosny; Ahmed, Hanaa Yousef \\ The Regional Center for Mycology \& Biotechnology, Al-Azhar University, Cairo, Egypt.
}

\begin{abstract}
Detoxification of mycotoxins must be taken to ensure food and feed safety. The aim of this study was to determine the influence of Yarrowia lipolytica on mycotoxin elimination and inhibition of Fusarium moniliforme growth. In this study, Y. lipolytica could remove 84.92\% of Fuminosin B1 (FB1) and 80.64\% of zearalenone (ZEN) from potato dextrose broth medium. Yeast cells and culture broth decreased FB1 by $34.16 \%$ and $82.59 \%$, along with decreasing ZEN by $51.83 \%$ and $75.34 \%$, respectively. Optimization results showed highest degradation percentage after incubation of $0.6 \mathrm{~g}$ yeast cells for $96 \mathrm{~h}$ at $37^{\circ} \mathrm{C}, \mathrm{pH} 6$ in the presence of glucose and peptone as $C$ and $N$ sources. Likewise, incubation with $\mathrm{Mg}^{2+}$ and $\mathrm{Se}^{2+}$ ions significantly increased toxin degradation. Proteinase K, EDTA and heat treatment decreased degradation activity, proving that the degradation is enzymatic. TEM observation confirmed the inhibitory effect of Yarrowia broth on $F$. moniliforme, indicating that inhibition of the toxin-producing strain is another mechanism of action. Conclusively, the applied yeast has detoxification capability of mycotoxins and especially FBI due to more than one mechanism. This study spotted the light on Yarrowia lipolytica strain that can be used to safely remove ZEN or FB1 toxicity from food and animal feed.
\end{abstract}

Keywords: Mycotoxins, detoxification, Fusarium moniliforme, Yarrowia lipolytica and TEM.

\section{Introduction}

Fusarium is a large cosmopolitan genus of imperfect fungi and it is of primarily interest because numerous species are important pathogens, produce a wide range of secondary metabolites, and/or cause opportunistic mycoses in humans and animals [1]. In recent years, mycotoxin contamination of cereal grain and animal feed with mycotoxins has become a global concern. It is estimated that it may affect as much as $25 \%$ of the world's food crops each year. Mycotoxins are toxic secondary metabolites produced by certain species of molds that commonly contaminate agricultural crops. The most common mycotoxins found in animal feed are aflatoxins, ochratoxins, trichothecenes, fumonisins, zearalenone and ergot alkaloids [2].

Currently, 28 structural fumonisin analogs are known, and the most abundant analogue in nature is fumonisin B1 (FB1), followed by fumonisin B2 (FB2) and fumonisin B3 (FB3) [3\&4].

Fumonisins are produced mainly by Fusarium verticillioides, and fumonisin contamination in corn has been observed in various areas of the world [5\&6]. FB1 has been implicated in disorders in animals such as leukoencephalomalacia in horses, pulmonary oedema syndrome in pigs, and showing nephrotoxicity, hepatotoxicity, and hepatocellular carcinogenicity in rats [7\&8].

The International Agency for Research on Cancer (IARC) classified FB1 as a probable human carcinogen (class 2B) [9]. To protect human health, the European Union (EU) has set a maximum level of $1000 \mathrm{mg} / \mathrm{kg}$ for the sum of FB1 and FB2 in corn and corn-based foods intended for direct human consumption [10].

Zearalenone (ZEN; 6-(10-hydroxy-6-oxo-trans-1-undecyl- $\beta$-resorcylic acid lactone) is a lactone derivative of resorcylic acid and a nonsteroidal estrogenic mycotoxin produced by several species belonging to the genus Fusarium [1]. High concentrations of ZEN are usually found in maize and hay stored under warm, humid conditions. However, ZEN is also a food contaminant, with concentrations as high as $289 \mu \mathrm{g} / \mathrm{g}$ [11\&12]. The estrogenic and possible carcinogenic effects of ZEN are of further concern [13-15] causing liver and kidney damage [16], endocrine disruption [17], as well as immunotoxicity were demonstrated [18]. These findings underline the urgent need to eliminate ZEN contamination in food and food products for human consumption. Because of the economic losses engendered by the mycotoxins and their negative impact on human and animal health, many strategies for detoxifying contaminated food and animal feed have been examined, including physical, chemical, and biological detoxification methods [19]. Of these, the biological control of Fusarium mycotoxins is an attractive alternative for efficiently eliminating toxins and thus safe-guarding the quality of food and feed [20]. Limitations such as loss of product nutritional and sensory qualities as well as the expensive equipment required for these techniques have encouraged the recent emphasis on biological methods [21]. Biological decontamination methods are being widely studied and may be a very promising choice, provided 
they show to be efficient, specific, cost-effective, and are environmentally friendly [22\&23].Yeast strains, due in large part to their GRAS (Generally Regarded As Safe) status and probiotic use, are of particular interest for reducing the bioavailability of mycotoxins [24]. However, a correlation between the $\beta$-D-glucan amount in the yeast cell wall and the efficacy of sequestering ZEN, aflatoxin B1, deoxinivalenol, patulin and ochratoxin A were demonstrated [25]. On the other hand, several studies report that some fungal, bacterial and yeast strains were able to in vitro biodegrade AFB1, OTA and ZEN [23, 26-28]. This fact suggests that more than one mechanism of mycotoxin clearance could be involved. The purpose of this study was to evaluate the ability of Yarrowia lipolytica strain, obtained from feedstuff, to remove fumonisin and zearalenone in vitro and the correlation between the factors affecting the mycotoxin reduction and yeast strain. Also, the possible mechanism of mycotoxin detoxification was studied.

\subsection{Tested strains}

\section{Materials And Methods}

Among thirty yeast strains from our laboratory collection used in biodetoxification screening experiments; Yarrowia lipolytica NRRL Y-1094 strain was then selected for studying possible detoxification mechanism. Fusarium moniliforme Sheldon ATCC 38932 stain was used as a mycotoxin producer strain. Yeast cells were cultivated in potato dextrose broth (PDB) medium for five days at $37^{\circ} \mathrm{C}$ together with mycotoxin standards. Potato dextrose broth (PDB) with tested mycotoxin without yeasts as well as individual yeast strains without mycotoxins in PDB were used as positive and negative controls.

\subsection{Chemicals:}

FB1 and ZEA standards were purchased from Sigma Aldrich (UK) and a stock solution was prepared at a concentration of $500 \mu \mathrm{g} / \mathrm{mL}$ in acetonitrile-water $(1: 1, \mathrm{v} / \mathrm{v}) . O$-phthaldialdehyde (OPA), 2-mercaptoethanol, sodium dihydrogenphosphate dihydrate, sodium tetraborate, hydrochloric acid, phosphoric acid, sodium chloride, disodium hydrogen phosphate, potassium dihydrogen phosphate and potassium chloride were of analytical grade from Merck (Darmstadt, Germany). Acetonitrile and methanol were of HPLC grade.

\subsection{Sample preparation:}

Treated and non-treated samples infected with Fusarium moniliforme were extracted using the method of Sydenham et al. [29] with some modifications. In brief, the samples (100ml) was extracted three times with acetonitrile/methanol/water $(25 / 25 / 50, \mathrm{v} / \mathrm{v} / \mathrm{v} ; 100 \mathrm{~mL})$ for $10 \mathrm{~min}$. each. After centrifugation, an aliquot of the supernatant was cleaned-up using anion extraction cartridges (Varian, Harbor City, CA, USA), which were preconditioned with methanol and water (3:1). After washing with methanol, the toxins were eluted with acetic acid-methanol $(1: 99, \mathrm{v} / \mathrm{v} ; 10 \mathrm{~mL})$, dried under nitrogen at $60^{\circ} \mathrm{C}$ and then stored at $4^{\circ} \mathrm{C}$ prior to analysis.

\subsection{Chromatographic conditions}

Dried samples were dissolved in methanol and an aliquot derivatized with o-phthaldialdehyde (OPA) for HPLC separation as previously reported [29\&30]. For OPA reagent preparation, $40 \mathrm{mg}$ of $O$ phthaldialdehyde was dissolved in $1 \mathrm{~mL}$ of methanol, and diluted with $5 \mathrm{~mL}$ of Na2B4O7 solution, then $50 \mu \mathrm{L}$ of 2-mercaptoethanol was added with gentle mixing. The formed mixture was stored in the dark in a capped amber vial. The reversed-phase HPLC separation was performed on a C18 column $(150 \times 4.60 \mathrm{~mm}$, Cosmosil 5 $\mathrm{C} 18-\mathrm{AR}, 5 \mu \mathrm{m})$. In case of FB1 detection, the column was eluted isocratically at a flow rate of $1 \mathrm{~mL} / \mathrm{min} \mathrm{with}$ methanol-0.1 M sodium dihydrogen phosphate (77:23; v/v) mobile phase adjusted to $\mathrm{pH} 3.35$ with o-phosphoric acid and optimal UV detection was obtained at $335 \mathrm{~nm}$. However, ZEN was separated with a mobile phase of water: acetonitrile $(50: 50, \mathrm{v} / \mathrm{v})$ at a flow rate of $1 \mathrm{ml} / \mathrm{min}$. and detection was carried out at $274 \mathrm{~nm}$ [31]. Injection volume was $20 \mu \mathrm{L}$. The HPLC instrument was configured with an Agilent (Waldbronn, Germany) 1100 series UV detector. Calibration was carried out in the range of 10-1000 $\mu \mathrm{g} / \mathrm{L}$. Data was collected and analyzed by Agilent ChemStation software and quantification was achieved by comparison of peak areas with those of authentic standards.

\subsection{Detoxification study in submerged fermentation:}

The culture medium containing the mycotoxin was inoculated with the yeast in a $150 \mathrm{~mL}$ flask. In parallel, non-contaminated and inoculated media (control 1) and contaminated but not inoculated media (control 2), were prepared. In all cases, triplicates were tested.Flasks containing the fermentative medium were inoculated for $240 \mathrm{~h}$, at $37{ }^{\circ} \mathrm{C}$ under orbital agitation $(200 \mathrm{rpm})$. A sample of fermented media $(5 \mathrm{~mL}) \mathrm{was}$ removed for analysis every $24 \mathrm{~h}$ and the residual mycotoxin was quantified. The sampling procedure was performed aseptically.

The percentage of mycotoxin degradation $(\mathrm{D} \%)$ was calculated using the following formula:

$D \%=(C 1-C 2) / C 3 \times 100 \%$

where: 
$C 1$ : The concentration of mycotoxin in the control after fermentation;

$C 2$ : The concentration of mycotoxin in treatment after fermentation;

C3: The initial concentration of mycotoxin before fermentation.

\subsection{Optimization of fermentation conditions:}

All optimization experiments were conducted in PDB. 24 h-old yeast-inoculum was inoculated in PDB $(100 \mathrm{~mL})$ containing mycotoxin $(5 \mu \mathrm{g} / \mathrm{mL})$ in a $250 \mathrm{~mL}$ flask with four replicates for each treatment. Inoculated cultures were incubated at $37{ }^{\circ} \mathrm{C}$ with agitation at $200 \mathrm{rpm}$ for $24 \mathrm{~h}$ in a shaker incubator. After incubation, the cells were removed by centrifugation at $10,000 \mathrm{~g}$ for $5 \mathrm{~min}$. The resulting supernatant was used for mycotoxin analysis. Sterile PDB was used to substitute microbial culture in the control. The mycotoxin degradation tests were performed as described in Section 2.5.

\subsubsection{Optimization of fermentation medium:}

Six culture media, including potato dextrose (PDB), Sabouraud dextrose (SDB), malt extract (MEB), yeast malt extract (YMB), yeast extract potato dextrose (YPD) and corn liquor broth media were screened to select the best fermentation medium for mycotoxin detoxification.For the carbon source tests, peptone was added as the nitrogen source during the tests. $20 \%$ glucose, galactose, arabinose, sucrose, maltose, mannitol, starch, lactose, ribose, trehalose, and fructose were individually introduced into PDB to determine the effect of the carbon source on mycotoxin degradation. The effect of different concentrations of the best carbon source on mycotoxin degradation was studied in PDB with $0.5 \%, 1 \%, 2 \%, 3 \%$, and $4 \%$ of optimized carbon source.

For the nitrogen source tests, the optimized carbon source was added as the carbon source. $0.5 \%$ peptone, beef extract, tryptone, sodium nitrate and ammonium chloride were individually introduced into the PDB medium to determine the effect of the nitrogen source on mycotoxin degradation. The effect of different concentrations of the best nitrogen source on mycotoxin degradation was also studied in PDB with $0.2 \%, 0.5 \%, 0.7 \%$, and $1 \%$ of optimized nitrogen source.

\subsubsection{Optimization of incubation temperature, period, amount of inoculum and $\mathrm{pH}$ :}

To measure the effect of temperatures, the reaction mixture was incubated at $10,20,30,37$, and $45^{\circ} \mathrm{C}$ with agitation at $200 \mathrm{rpm}$ for $96 \mathrm{~h}$ in a shaker incubator. Different incubation periods $(1,4,12,24,48,72,96$, 120,168 and $240 \mathrm{~h}$ ) were also tested to explore the mycotoxin detoxification kinetics.

In the $\mathrm{pH}$ tests, initial $\mathrm{pH}$ value in culture medium was adjusted to $3,4,5$ and 6 by using $\mathrm{HCl}$, and to 7, 8, 9, and 10 by using $\mathrm{NaOH}$.

To test the effect of the amount of inoculum, the cell pellets were washed three times with PBS, then $0.2,0.4$, $0.6,0.8$ and $1.0 \mathrm{~g}$ pellets were suspended in $9 \mathrm{~mL}$ PBS solution infused with $1 \mathrm{~mL}, 50 \mu \mathrm{g} / \mathrm{mLZEN}$ or FB1, and incubated at $37^{\circ} \mathrm{C}, 200 \mathrm{rpm}$ under aerobic conditions for $96 \mathrm{~h}$, respectively.

To evaluate the effect of different metal ions, $\mathrm{Fe}^{3+}, \mathrm{Mg}^{2+}, \mathrm{Zn}^{2+}, \mathrm{Cu}^{2+}, \mathrm{Mn}^{2+}, \mathrm{Se}^{2+}$ and $\mathrm{Li}^{+}$(in the form of $\mathrm{FeCl}_{3}$, $\mathrm{MgSO}_{4}, \mathrm{ZnCl}_{2}, \mathrm{CuSO}_{4}, \mathrm{MnCl}_{2}, \mathrm{SeCl}_{2}$, and $\mathrm{LiCl}$ ) in a concentration of $5 \mathrm{mM}$ was separately added to the reaction mixture. The reaction mixture was incubated at $37{ }^{\circ} \mathrm{C}$ with agitation at $200 \mathrm{rpm}$ for $96 \mathrm{~h}$ in a shaker incubator.

\subsubsection{Degradation of mycotoxin by the culture broth, cells and cell extracts:}

To identify the mechanism by which $Y$. lipolytica strain exhibited detoxification activity, the cells, cell extract, and culture broth were assayed in separate experiments as described before [21, 23]. The yeast cells were separated from the broth medium by centrifugation at 10,000 $\mathrm{xg}$ for 5 min., after which the culture broth was sterilized using $0.2-\mu \mathrm{m}$ disposable syringe filters (Millipore, Bedford, MA, USA). The yeast cells were then divided into two portions based on treatment either viable cells or autoclaved cells $\left(121^{\circ} \mathrm{C}\right.$ for $20 \mathrm{~min}$.).The cell extracts were prepared by the following procedures; the cell pellets were resuspended in phosphate buffered saline ( $\mathrm{pH}$ 7.4) in preparation for cell rupture. The suspension was disintegrated thrice on ice by using a Virsonic ultrasonic cell disintegrator (Virtis, USA). The disintegrated cell suspension was centrifuged at 12,000 $x g$ for $20 \mathrm{~min}$ at $4{ }^{\circ} \mathrm{C}$, and then the supernatant was filtered aseptically using sterile $0.2 \mu \mathrm{m}$ cellulose pyrogen free filters. The degradation tests were carried out after incubation at $37^{\circ} \mathrm{C}$ with agitation at $200 \mathrm{rpm}$ for $72 \mathrm{~h}$ in a shaker incubator as described in 2.5. PDB was used to substitute supernatant in the control. The phosphate buffered saline was used to substitute cell and cell extracts in the control.

\subsubsection{Effects of protease, heat and EDTA on zearalenone degradation activity in culture broth:}

In order to study the factors affecting the degradation activity, the culture broth were treated separately with $1 \mathrm{mg} / \mathrm{ml}$ proteinase $\mathrm{K}$ plus $1 \%$ SDS for $1 \mathrm{~h}$ at $100{ }^{\circ} \mathrm{C}$ water for $10 \mathrm{~min}$ and $0.1 \mathrm{M}$ EDTA [33]. The effect of heat treatment was also determined by dipping the culture broth at 50 and $80^{\circ} \mathrm{C}$ for 2,4 , and $6 \mathrm{~h}$, respectively. The culture broth was included as negative control; however, sterile PDB media with ZEN or FB1 were included as blank control. ZEN or FB1 was added to all of the preparations described in previous sections, to a final concentration of $5 \mu \mathrm{g} / \mathrm{mL}$. All of the samples were incubated at $200 \mathrm{rpm}$ at $37^{\circ} \mathrm{C}$ under for $96 \mathrm{~h}$. 


\subsection{Antifungal activity of yeast metabolites:}

The antifungal activity of extracellular metabolites produced from Yarrowia lipolytica was investigated against Fusarium moniliforme using agar well diffusion method with slight modifications [34]. Briefly, Fusarium moniliforme culture was spread onto solidified MEA media in Petri-dish (150 mm x $20 \mathrm{~mm}$ ). The plate was then punched with a $6 \mathrm{~mm}$ diameter cork-borer to create wells. The yeast metabolites were added into each well. The plates were first incubated for $2 \mathrm{~h}$ at $4 \pm 2{ }^{\circ} \mathrm{C}$ followed by $48 \mathrm{~h}$ at $28 \pm 2{ }^{\circ} \mathrm{C}$. Then, the antimicrobial activity was determined by measuring the mean diameters of inhibition zone in millimeter.

\subsection{TEM observations of treated cells:}

The treated and untreated Fusarium moniliforme cells were observed under Transmission Electron Microscope (TEM). The samples were prepared by standard protocol. Samples were fixed in 1\% Glutaraldyhde then washed in $0.1 \mathrm{M}$ buffer, $1 \%$ Osmium tetroxide was used for post-fixation and again washed with $0.1 \mathrm{M}$ buffer. The samples were dehydrated in a series of ethanol and acetone, infiltrated, embedded in epoxy EMbed 812 resin and sectioned using Leica Ultracut ultramicrotome. These ultra-thin sections (60 nm) were cut and placed on copper grids, counterstained with saturated uranyl acetate and aqueous lead citrate. Finally, the grids were dried in a desiccator and examined at $80 \mathrm{kV}$ using TEM (JEOL-JEM 1010, Japan) at the Regional Center for Mycology and Biotechnology (RCMB), Al-Azhar University, to study the action of yeast metabolites on fungal cells and any morphological changes.

\subsection{Statistical Analysis:}

All specific experiments were repeated three times. Statistical analysis was performed using Statistix 8.1 (Analytical Software, Tallahassee, FL, USA, 2005).

\subsection{Degradation of FB1 and ZEN by the culture broth, cells and cell extracts:}

With the growing frequency of food and feed safety issues, there has been an increased focus, specifically, on bio-pollution problems, in which mycotoxins are one of the main factors causing food and feed contamination. In this study, thirty yeast strains were screened for biological detoxification screening capabilities; the screening experiments showed that Yarrowia lipolytica NRRL Y-1094 strain exhibited the highest ability to reduce mycotoxins produced by Fusarium moniliforme Sheldon ATCC 38932.

The FB1 and ZEN degradation activity of $Y$. lipolytica culture broth was significantly stronger than cells and cell extracts (Fig. 1). Moreover, the dead cells (autoclaved) exhibited the lowest detoxification activity against the two mycotoxins (Fig. 1). The culture broth, viable cells, autoclaved cells and cell extracts of $Y$. lipolytica could degrade $82.59 \%, 34.16 \%, 13.82 \%$ and $25.89 \% \mathrm{FB} 1$, as well as $75.34 \%, 51.83 \%, 20.29 \%$ and $18.41 \%$ ZEN, respectively. Some similar results were reported. Culture supernatant of Stenotrophomonas maltophilia was able to degrade $78.7 \%$ mycotoxin after $72 \mathrm{~h}$ incubation [35]. The extracellular extracts of Rhodococcus erythropolis liquid culture could degrade $66.8 \%$ aflatoxin B1 after $72 \mathrm{~h}$ incubation [36]. In addition, the active ingredient in the culture supernatant was considered to be enzymatic [36].

\subsection{Optimization of fermentation conditions for FB1 and ZEN degradation:}

In this study, Yarrowia lipolytica could remove $84.92 \%$ of fuminosin B and $80.64 \%$ of zearalenone from potato dextrose broth medium. Lower detoxification percentages were detected by the other tested culture media (Fig. 2). These detoxification levels were increased with optimization of the fermentation conditions.

The effect of different incubation times on the FB1 and ZEN degradation activity of Y. lipolytica was also tested. The degradation process by $Y$. lipolytica was relatively fast and continuous, with 9 and $15.4 \%$ of FB1 and ZEN removed in the first $1 \mathrm{~h}, 43.9$ and 36.8\% removed after $12 \mathrm{~h}, 54.6$ and $44.5 \%$ removed after $24 \mathrm{~h}, 70.8$ and $62.9 \%$ removed after 48 h, 82.6 and $75.3 \%$ removed after 72 h, 90.76 and $84.2 \%$ removed after 96 h (Fig. 3). Non-significant difference in the degradation of FB1 and ZEN could be detected after further increase in incubation time from 96 to $240 \mathrm{~h}$. Alberts et al. reported that a significant $(p<0.05)$ reduction in aflatoxin B1 was already observed after $2 \mathrm{~h}$ in the presence of Rhodococcus erythropolis extracellular extracts with only $33.2 \%$ residual aflatoxin $\mathrm{B} 1$ after $72 \mathrm{~h}$ [36]. Additionally, Hormisch et al. found that the aflatoxin B1 concentration was reduced to amounts of $70 \%$ to $80 \%$ of the initial concentration within 36 h by Mycobacterium fluoranthenivorans [37].

When using carbon sources like glucose, galactose, arabinose, sucrose, maltose, mannitol, starch, lactose, ribose, trehalose, and fructose, the degradation in the presence of glucose was significantly higher than other carbon sources (Fig. 4). Moreover, the detoxification process increased with the increased concentration of glucose. Among the five different concentrations of glucose tested, 4\% was the best for ZEN degradation; however, 3\% was the best for FB1 degradation (Fig. 5). Different carbon sources affect the growth of microorganisms. The growth of microorganisms was stimulated when the carbon source was utilizable [38]. 
When the carbon source was difficult to be utilized, the microorganism strains tended to utilize other carbon sources, which might promote a decrease in other carbon-based compounds [39,40]. As shown in Fig. 6, when the nitrogen source was tested, peptone exhibited significant higher effect on the detoxification process compared with the other $\mathrm{N}$ sources (beef extract, tryptone, ammonium salt and sodium nitrate). Among the four different concentrations of peptone tested, $0.5 \%$ was the most suitable for both mycotoxins degradation (Fig. 7). Appropriate nitrogen source and concentration would not only stimulate the growth but also affect the expression of biosynthetic genes and therefore the production of microorganisms [40-42].

The effect of temperature on FB1 and ZEN degradation by the culture broth of $Y$. lipolytica is shown in Fig. 8. The highest percentage of degradation was obtained at $37{ }^{\circ} \mathrm{C}$. The temperature affects the growth of microorganisms, thus the metabolic production. The initial $\mathrm{pH}$ value in PDB showed a significant effect on FB1 and ZEN degradation (Fig. 9). In the present study, the highest percentage of degradation was obtained at pH 6 and lowest at $\mathrm{pH} 3$ and 10. The $\mathrm{pH}$ of medium is a very important environmental factor, which is often neglected. Many investigators claimed that the different morphology of fungal mycelia under different initial $\mathrm{pH}$ values was the critical factor in biomass accumulation and metabolite formation $[43,44]$. The correlation of FB1 and $\mathrm{ZEN}$ degradation with $\mathrm{pH}$ values is typical for enzymatic reactions.

The effect of amount of inoculum on FB1 and ZEN degradation was also analyzed. The optimal fermentation conditions observed with the amount of inoculum of $0.6 \mathrm{~g}$, inoculated cultures when incubated at $37{ }^{\circ} \mathrm{C}$ for $96 \mathrm{~h}$ in a shaker incubator (Fig. 10). It has been reported previously that growth and enzyme production of microbes were affected by variations in incubation temperature, period and amount of inoculum $[21,45]$.

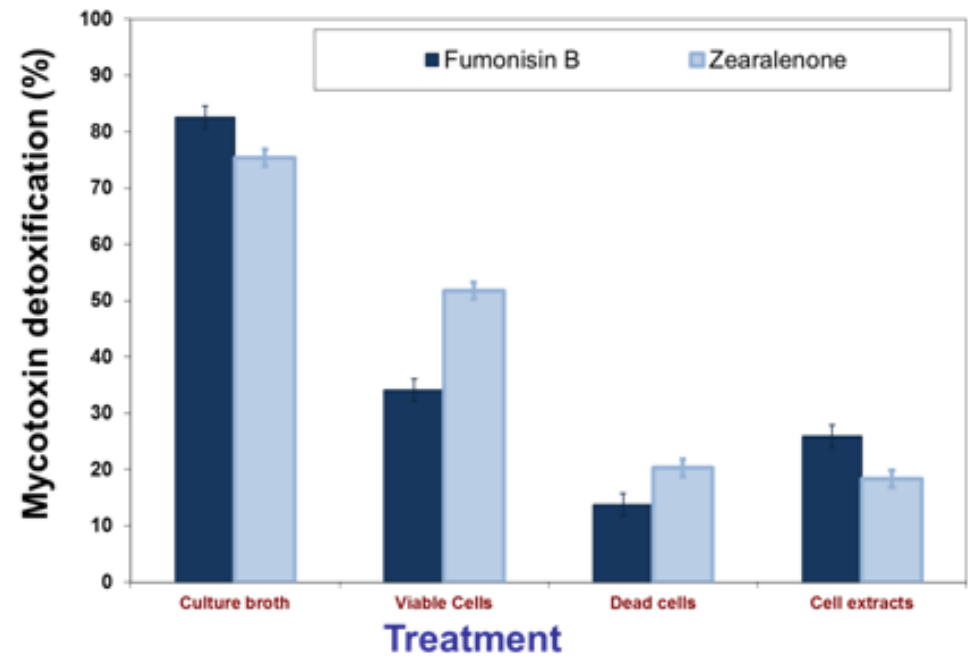

Figure1. Detoxification of the mycotoxin by the culture broth, cells and cell extracts of Yarrowia lipolytica after $72 \mathrm{~h}$ incubation. The medium was used to substitute culture broth in the control. The phosphate buffered saline was used to substitute cells and cell extracts in the control. Results are expressed as means \pm SEM from three separate experiments.

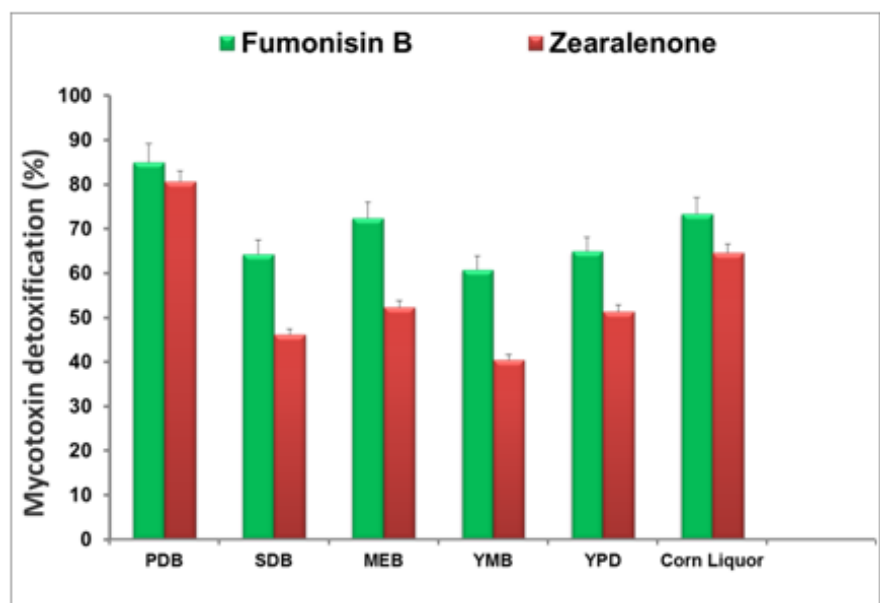

Figure 2. Effect of different culture media on the mycotoxin detoxification by Yarrowia lipolytica after $96 \mathrm{~h}$ incubation. 

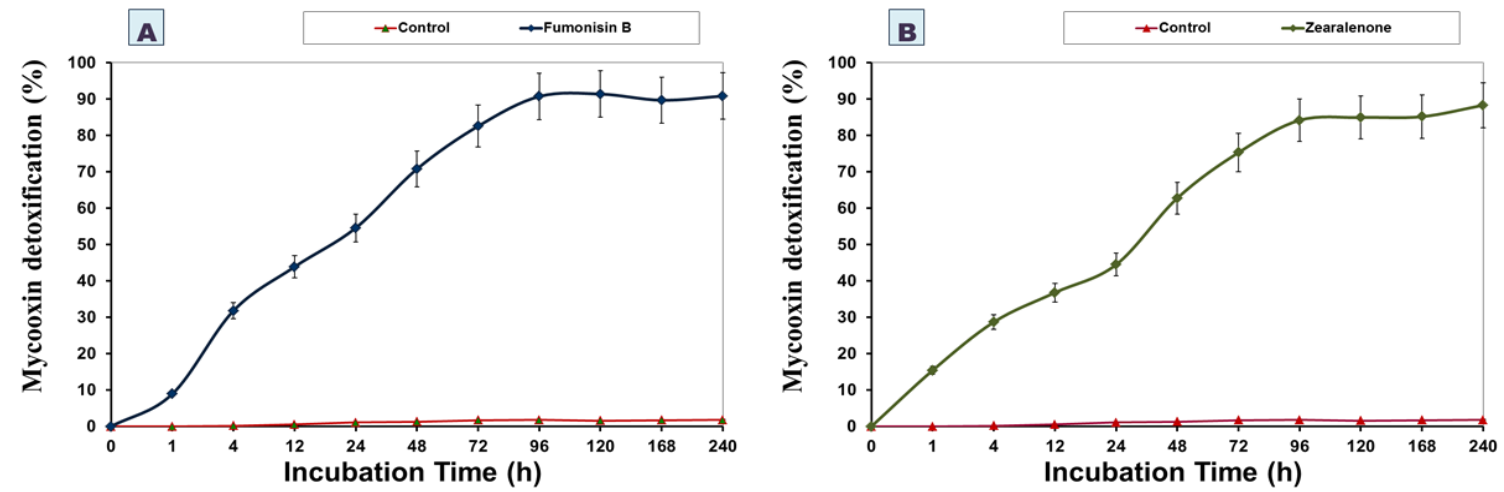

Figure 3. Kinetics of mycotoxin detoxification during $240 \mathrm{~h}$ of fermentation by Yarrowia lipolytica showing the effect of different incubation periods on the detoxification process of fumonisin B (A) and zearalenone (B).

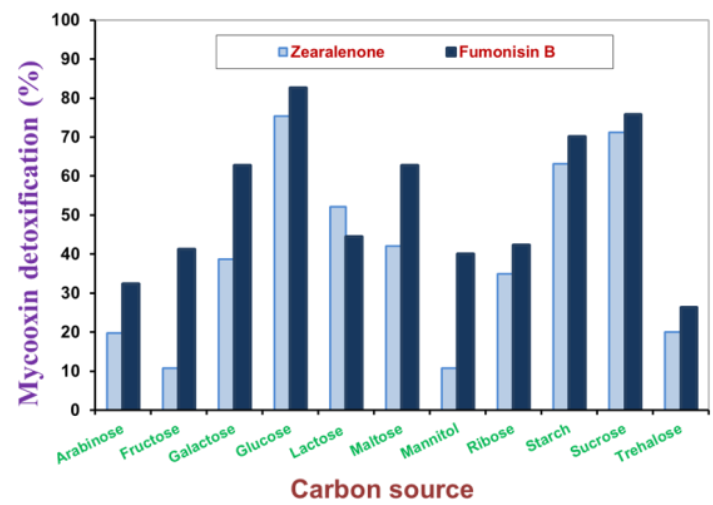

Figure 4. Effect of different carbon sources on the mycotoxin detoxification by Yarrowia lipolytica after $96 \mathrm{~h}$ incubation.

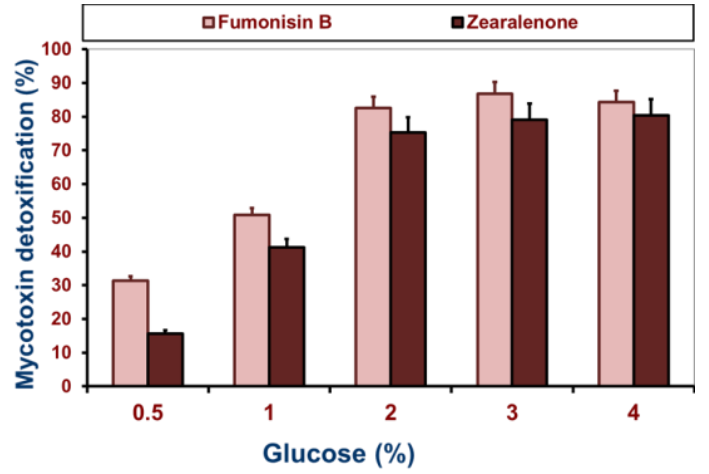

Figure 5. Effect of different glucose contents on the mycotoxin detoxification by Yarrowia lipolytica after $96 \mathrm{~h}$ incubation.

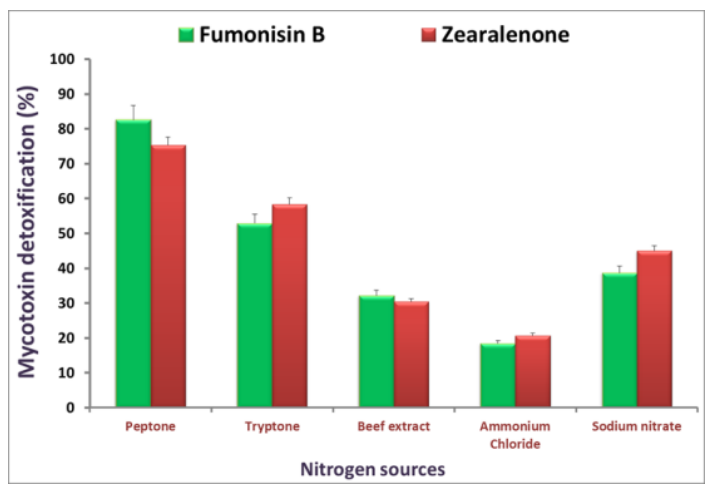

Figure 6. Effect of different nitrogen sources on the mycotoxin detoxification by Yarrowia lipolytica after $96 \mathrm{~h}$ incubation. 


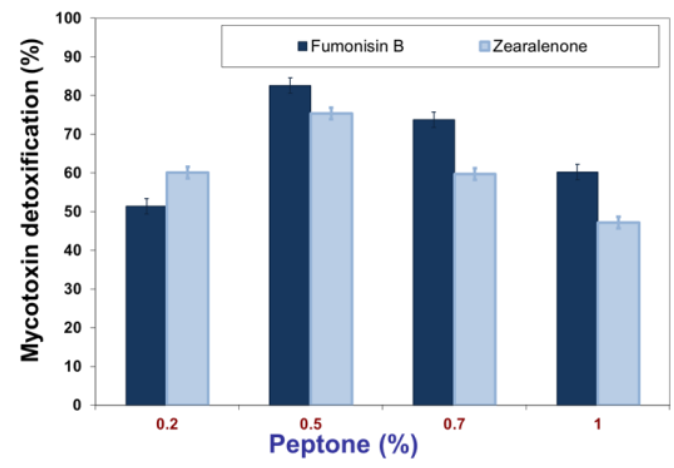

Figure 7. Effect of different peptone contents on the mycotoxin detoxification by Yarrowia lipolytica after $96 \mathrm{~h}$ incubation.

The effect of different metal ions on FB1 and ZEN degradation is shown in Fig. 11. Compared to the treatment without ions, $\mathrm{Li}^{+}$and $\mathrm{Zn}^{2+}$ showed the strongest inhibition function $(p<0.001)$ in mycotoxin detoxification process. Likewise, incubation with $\mathrm{Se}^{2+}$ and $\mathrm{Mg}^{2+}$ significantly increased toxin degradation. $\mathrm{Fe}^{3+}$, $\mathrm{Cu}^{2+} \& \mathrm{Mn}^{2+}$ differed in their effect (Fig. 11). The effects of metal ions on degradation activity further supported the enzyme involvement in mycotoxin degradation by the isolate. Also, the correlation of mycotoxin degradation with $\mathrm{pH}$ values is typical for enzymatic reactions. Similar results were reported that $\mathrm{Zn}^{2+}, \mathrm{Cu}^{2+}$, and $\mathrm{Mn}^{2+}$ inhibited aflatoxin B1 degradation by Flavobacterium aurantiacum [46, 47].

\subsection{Possible mechanism of FB1 and ZEN detoxification:}

However, enzymatic effects in culture broth were suggested as the possible mechanism by which significantly degraded the mycotoxin more than $80 \%$. The percentage of mycotoxin degradation decreased when the culture broth dipped at 50 and $80{ }^{\circ} \mathrm{C}$ for $6 \mathrm{~h}$ (Fig. 12). Heat treatment decreased the FB1 and ZEN degradation activities of yeast culture broth. The higher the temperature was, the faster the activity decreased. Similarly, Guan et al. reported that when culture supernatant was heated (boiling water bath for $10 \mathrm{~min}$ ), no mycotoxin degradation activity was observed [35].

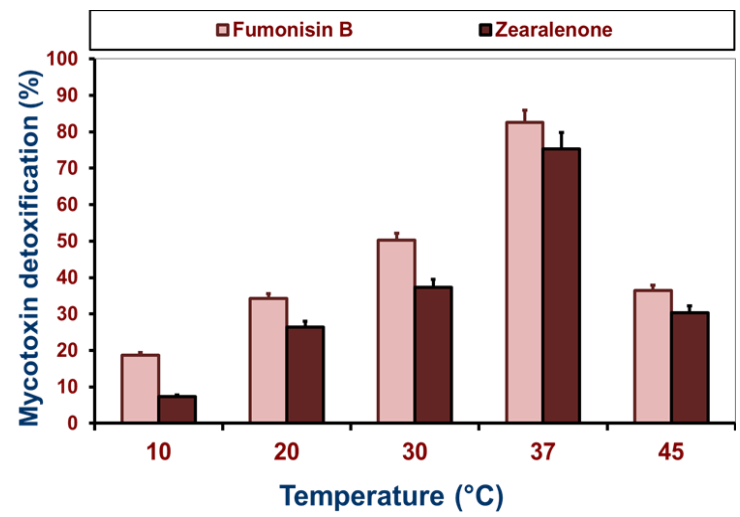

Figure 8. Effect of different temperature on the mycotoxin detoxification by Yarrowia lipolytica after $96 \mathrm{~h}$ incubation.

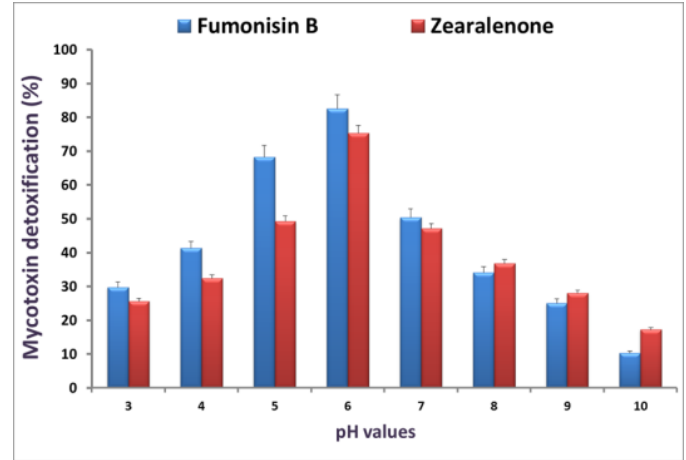

Figure 9. Effect of different $\mathrm{pH}$ values on the mycotoxin detoxification by Yarrowia lipolytica after $96 \mathrm{~h}$ incubation. 


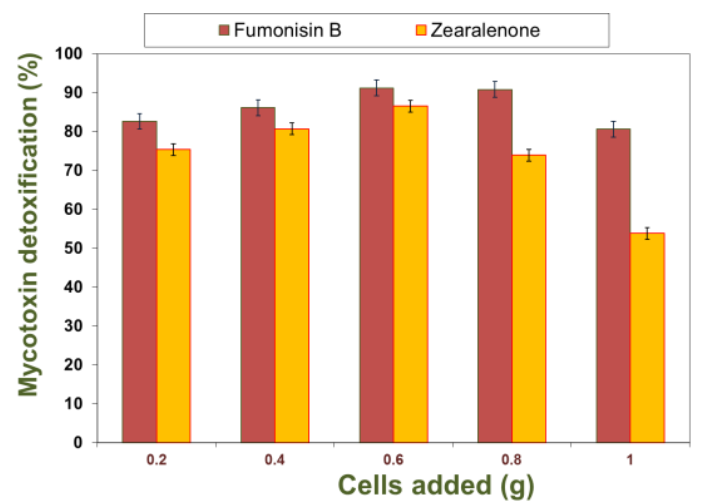

Figure 10. Effect of cell amounts on the mycotoxin detoxification by Yarrowia lipolytica after $96 \mathrm{~h}$ incubation.

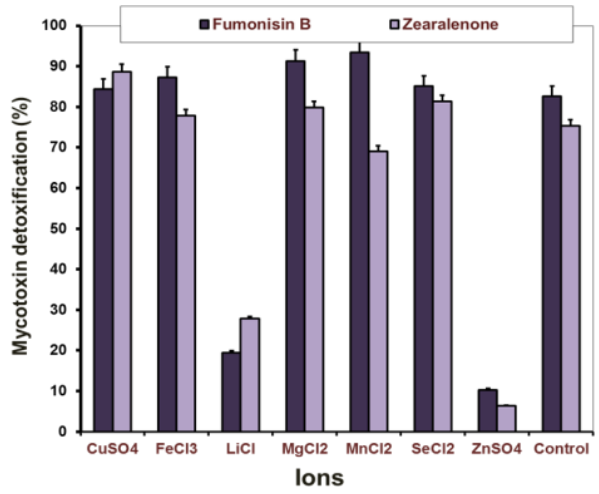

Figure 11. Effect of metal ions on the mycotoxin detoxification by Yarrowia lipolytica after $96 \mathrm{~h}$ incubation.

After treatment with proteinase $\mathrm{K}$ and heat (Fig. 12\&13), the pooled culture broth retained low mycotoxin degradation activity, which indicated that enzymes from the pooled active culture broth are involved in the degradation. EDTA, as metal chelating agent, can destroy the FB1 and ZEN degradation activity (Fig. 13), which indicated that some metal cations are required for the enzymes in the active fractions to degrade FB 1 and ZEN as previously reported [47]. All indications are that an extracellular metalloenzyme is responsible for the degradation by culture broth. Moreover, incubation of the toxin with either heat-treated or viable cells resulted in lower percentage of ZEN removal from the medium, indicating that binding, not metabolism, was the removal mechanism by the cells. The reason is that both adsorption and degradation effects exist in the yeast suspension.

The antifungal activity of the culture broth produced from Yarrowia lipolytica was observed against Fusarium moniliforme using agar well diffusion method (Fig. 14). Transmission electron microscopic study confirmed the inhibitory effect of Yarrowia ipolytica broth on Fusarium moniliforme, and indicated that the mycelium had lost its cytoplasmic contents and became highly vacuolated compared to untreated mycelia (Fig. 15). Similar reports $[11,28]$ showing the inhibition of toxin and toxin-producing strains are in the same line of the current study. Previously, Molnar described a new yeast strain, Trichosporon mycotoxinivorans, which is able to degrade ZEN to carbon oxide and other non-toxic metabolites [48]. Takahashi-Ando characterized a novel lactonohydolase enzyme from the fungus Clonostachys rosea which convert ZEN to a less toxic metabolite [26]. Several Rhizopus strains, including $R$. stolonifer, $R$. oryzae and $R$. microsporus, were found to completely degrade ZEN [49]. Similarly, Rhodococcus pyridinivorans, Bacillus, and Lactobacillus strains were proved to be very efficient to eliminate ZEN in culture media [50-52].

Products containing yeast materials have potential to adsorb mycotoxins due to the physical properties of the yeast cell wall, which has structures that allow for binding of mycotoxins $[53,54]$. The $\beta$-glucan of yeast can from single or triple helix polysaccharide chains which can give adsorptive capacities for mycotoxins through hydrogen and ionic bonding, and van der Waals interactions [55]. Previous in vitro studies have shown that $\beta$-glucan isolated from yeast cell walls has the capacity to adsorb up to 50\% of ZEA [56]. The yeast fermentation product may also play a role in adsorbing mycotoxins, due to the fact that it contains $\beta$-glucan. Together, these effects of the yeast fermentation product may improve the ability of the animal to fight mycotoxins [24, 57]. 

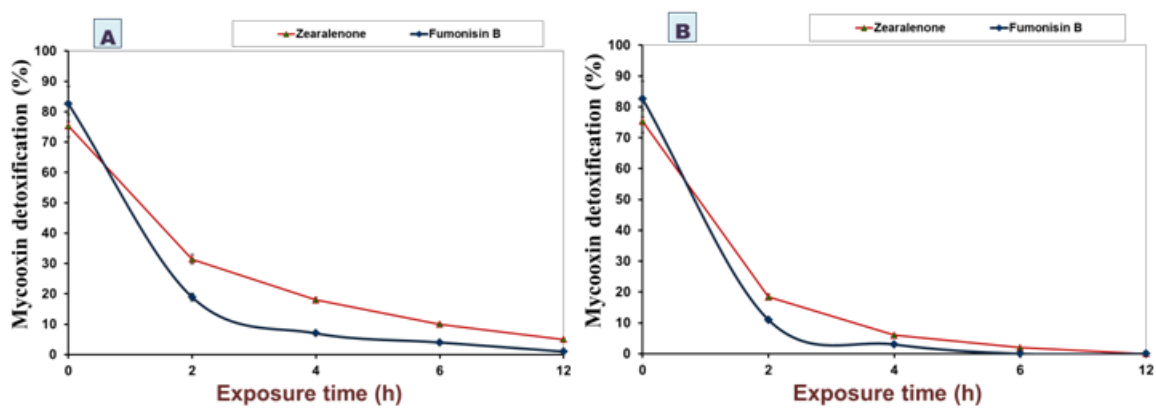

Figure 12. Effect of heat treatment on mycotoxin detoxification during $12 \mathrm{~h}$ of fermentation by Y. lipolytica showing the effect of incubation at $50{ }^{\circ} \mathrm{C}(\mathrm{A})$ and $80^{\circ} \mathrm{C}(\mathrm{B})$ on the detoxification process.

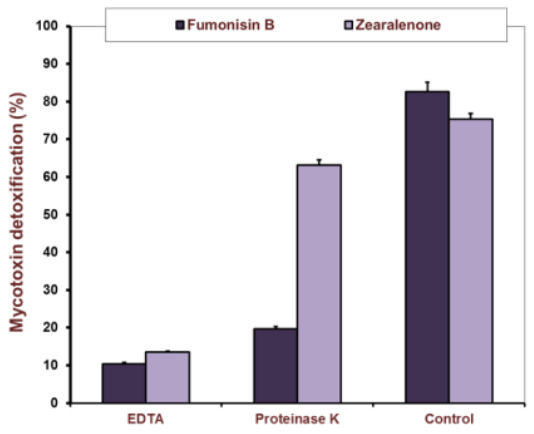

Figure 13. Effect of proteinase $K$ and EDTA treatment on mycotoxin detoxification on the detoxification process.

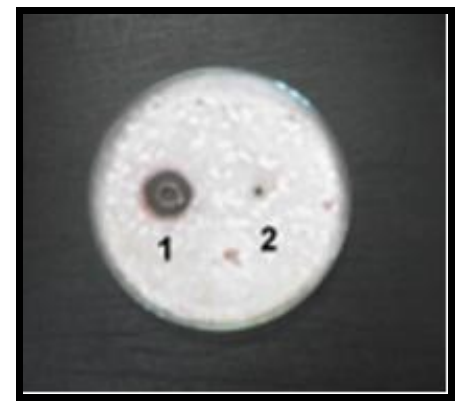

Figure 14. The inhibitory effect of Yarrowia lipolytica extracellular metabolites (broth) on growth of Fusarium moniliforme showing the inhibition zones in response to yeast metabolites (1) and no inhibitory effect on cell extract (2).
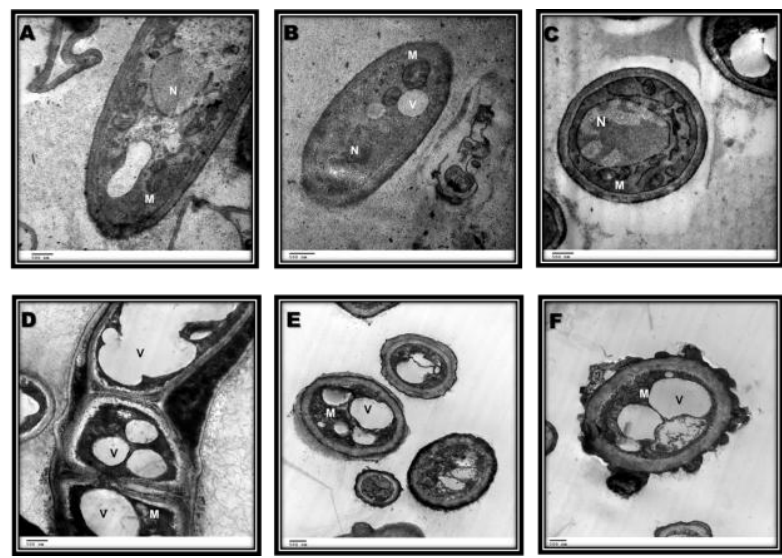

Figure 15. Transmission Electron Micrographs representing morphological changes in Fusarium moniliforme treated with Yarrowia lipolytica. A-C: Fusarium moniliforme control showing normal mycelium and conidium. D-F: Fusarium moniliforme treated with Y. lipolytica extracellular metabolites (broth) showing highly vacuolated mycelium. (where, M: Mitochondria, N: nucleus, V: vacuole); scale bar $=500 \mathrm{~nm}$. 


\section{Conclusions}

In this study, the environmentally friendly Yarrowia lipolytica NRRL Y-1094 strain reduces FB1 and ZEN contamination as well, via mechanisms involving binding and metabolism of the toxin along with inhibitory effect on the toxin-producing strain Fusarium moniliforme, thereby alleviating hazards to human and animal health. In the culture broth, the activities of extracellular enzymes were most likely responsible for FB1 and ZEN degradation. This ability to remove the toxin was greater in yeast exponential growth phase, and that the process was a quick one, being saturated after 1 hour of contact. The great advantage in the commercial use of this yeast as detoxification agent is that it is already used in a wide range of fermented food products, being recognized as safe. Therefore, we suggest that Yarrowia lipolytica NRRL Y-1094 strain can be used to safely remove ZEN or FB1 toxicity from food and animal feed. Functional and technological tests should be performed to validate in vivo efficiency.

\section{Conflict of Interest}

The authors declare that there are no conflicts of interest.

\section{References}

[1]. T. Tanaka, A. Hasegawa, S. Yamamoto, U.S. Lee, Y. Sugiura, Y. Ueno, Worldwide contamination of cereals by the Fusarium mycotoxins nivalenol, deoxynivalenol, and zearalenone. J. Agric. Food Chem. 36, 1988, 979-983.

[2]. V. Aiko, A. Mehta, Occurrence, detection and detoxification of mycotoxins. J. Biosci. 40, 2015, 943-954.

[3]. J.P. Rheeder, W.F. Marasas, H.F. Vismer, Production of fumonisin analogs by Fusarium species. Appl. Environ. Microbiol., 68, 2002, 2101-2105.

[4]. M. Kushiro, K. Tanaka, S. Miyazaki, T. Nagata, Advances of liquid chromatographic determination of fumonisin; potential mycotoxins for humans, Curr. Pharm. Anal., 2, 2006, 289-297.

[5]. Joint FAO /WHO Expert Committee on Food Additives. Safety evaluation of certain food additives and contaminants in food: fumonisins, Proc. $56^{\text {th }}$ Meeting of the Joint FAO/WHO Expert Committee on Food Additives. World Health Organization, Geneva, 2001, 103-279.

[6]. W.F. Marasas, Discovery and occurrence of the fumonisins: a historical perspective. Environ. Health Perspect. 109, 2001, 239-243.

[7]. WHO. Environmental health criteria 219: Fumonisin B1. Geneva, 2000.

[8]. K.R.N. Reddy, B. Salleh, B. Saad, H.K. Abbas, CA. Abel, WT. Sheir, An overview of mycotoxin contamination in foods and its implications for human health. Toxin Rev. 29, 2010, 3-26.

[9]. International Agency for Research on Cancer: Fumonisin B1. IARC Monographs on the evaluation of carcinogenic risks to humans, IARC, Lyons, France: IARC Press., 82, 2002, 301-366.

[10]. European Commission. Regulation (EC) No 1126/2007 setting maximum levels for certain contaminants in foodstuffs as regards Fusarium toxins in maize and maize products. Off J Eur Union. L. 255, 2007, 14-17.

[11]. J.C. Kim, H. Kang, D.-H. Lee, Y. Lee, T. Yoshizawa, Natural occurrence of Fusarium mycotoxins (trichothecenes and zearalenone) in barley and corn in Korea. Appl. Environ. Microbiol. 59, 1993, 3798-3802.

[12]. K.E. Yuwai, K.S. Rao, K. Singh, T. Tanaka, Y. Ueno, Occurrence of nivalenol, deoxynivalenol, and zearalenone in imported cereals in Papua, New Guinea. Nat. Toxins 2, 1994, 19-21.

[13]. V. Ehrlich, F. Darroudi, M. Uhl, H. Steinkellner, M. Gann, B. Majer, M. Eisenbauer, S. Knasmuller, Genotoxic effects of ochratoxin A in human-derived hepatoma (HepG2) cells. Food Chem. Toxicol. 40, 2002, 1085-1090.

[14]. M. Metzler, E. Pfeiffer, A. Hildebrand, Zearalenone and its metabolites as endocrine disrupting chemicals. World Mycotoxin J. 3 , $2010,385-401$.

[15]. X. Sun, X. He, K. Xue, Y. Li, D. Xu, H. Qian, Biological detoxification of zearalenone by Aspergillus niger strain FS10. Food and Chemical Toxicology, 72, 2014, 76-82.

[16]. E. Conkova, A. Laciakova, B. Pastorova, H. Seidel, G. Kovac, The effect of zearalenone on some enzymatic parameters in rabbits. Toxicol. Lett. 121, 2001, 145-149.

[17]. C.L. Hughes Jr., M.M. Chakinala, S.G. Reece, R.N. Miller, D.W. Schomberg Jr., K.B. Basham, Acute and subacute effects of naturally occurring estrogens on luteinizing hormone secretion in the ovariectomized rat: Part 2. Reprod. Toxicol. 5, 1991, $133-137$.

[18]. L. Berek, I. Petri, A. Mesterhazy, J. Teren, J. Molnar, Effects of mycotoxins on human immune functions in vitro. Toxicol. In vitro $15,2001,25-30$

[19]. M.R. Armando, R.P. Pizzolitto, C.A. Dogi, A. Cristofolini, C. Merkis, V. Poloni, A.M. Dalcero, L.R. Cavaglieri, Adsorption of ochratoxin A and zearalenone by potential probiotic Saccharomyces cerevisiae strains and its relation with cell wall thickness. Journal of Applied Microbiology 113, 2012, 256-264.

[20]. A.D. Altalhi, Plasmid-mediated detoxification of mycotoxin zearalenone in Pseudomonas sp. ZEA-1. Am. J. Biochem. Biotechnol. 3, , 2007.

[21]. O. Teniola, P. Addo, I. Brost, P. Farber, K.-D. Jany, J. Alberts, W. Van Zyl, P. Steyn, W. Holzapfel, Degradation of aflatoxin B1 by cell-free extracts of Rhodococcus erythropolis and Mycobacterium fluoranthenivorans sp. nov. DSM44556T. Int. J. Food Microbiol. 105, 2005, 111-117.

[22]. A. Bata, R. Lasztity, Detoxification of mycotoxin-contaminated food and feed by microorganisms. Trends Food Sci. Technol. 10, 1999, 223-228.

[23]. M.M. Elaasser, R.A. El Kassas, Detoxification of aflatoxin B-1 by certain bacterial species isolated from Egyptian soil. World Mycotoxin Journal; 4(2), 2011, DOI:10.3920/WMJ2010.1262

[24]. A.C. Weaver, M.T. See, S.W. Kim, Protective effect of two yeast based feed additives on pigs chronically exposed to deoxynivalenol and zearalenone. Toxins, 6, 2014, 3336-3353.

[25]. A. Yiannikouris, G. Andre, A. Buleon, G. Jeminet, I. Canet, J. Francois, G. Bertin, J.P. Jouany, Comprehensive conformational study of key interactions involved in zearalenone complexation with beta-D-glucans. Biomacromolecules 5, 2004, 2176-2185.

[26]. N. Takahashi-Ando, T. Tokai, H. Hamamoto, I. Yamaguchi, M. Kimura, Efficient decontamination of zearalenone, the mycotoxin of cereal pathogen, by transgenic yeasts through the expression of a synthetic lactonohydrolase gene. Appl Microbiol Biotechnol., $67,2005,838-844$

[27]. J. Biernasiak, M. Piotrowska , Z. Libudzisz, Detoxification of mycotoxins by probiotic preparation for broiler chickens. Mycotoxin Res.; 22(4), 2006, 230-5. doi: 10.1007/BFO2946747. 
[28]. H.H. El-Shiekh, H.M. Mahdy, M.M. Elaasser, Bioremediation of Aflatoxins by Some Reference Fungal Strains. Polish Journal of Microbiology, 56 (3), 2006, 215-223.

[29]. E.W. Sydenham, GS Shephard, PG. Thiel, S. Stockenström, PW. Snijman, DJ. Van Schalkwyk, Liquid chromatographic determination of fumonisins B1, B2, and B3 in corn: IUPAC/AOAC interlaboratory collaborative study. J AOAC Int 79, 1996, 688696.

[30]. N. Ndube, L. van der Westhuizen, G.S. Shephard, Determination of fumonisins in maize by HPLC with ultraviolet detection of $o$ phthaldialdehyde derivatives. Mycotox Res., 25, 2009, 225-228.

[31]. T.B. Whitaker, A.B. Slate, A.S. Johansson, Sampling feeds for mycotoxin analysis, in D.Diaz (Ed.), The Mycotoxin Blue Book, Nottingham University Press: Bath, UK, 2005, 1-13.

[32]. K. Cho, J. Kang, W. Cho, C. Lee, J. Ha, K.B. Song, In vitro degradation of zearalenone by Bacillus subtilis. Biotechnol. Lett. 32, 2010, 1921-1924.

[33]. Y. Yu, L. Qiu, H. Wu, Y. Tang, Y. Yu, X. Li, D. Liu, Degradation of zearalenone by the extracellular extracts of Acinetobacter sp. SM04 liquid cultures. Biodegradation 22, 2011, 613-622.

[34]. H.H. ElSheikh, M.M. Elaasser, H.M. Magdy, S.M. Abdel-Kareem, Antimicrobial, antitumor and antioxidant activities of certain marine fungi isolated from Alexandria. Afr. J. Mycol. \& Biotech. 19, 2014, 13-22.

[35]. S. Guan, C. Ji, T. Zhou, J. Li, Q. Ma, T. Niu, Aflatoxin B1 degradation by Stenotrophomonas maltophilia and other microbes selected using coumarin medium. Int. J. Mol. Sci. 9, 2008, 1489-1503.

[36]. J.F. Alberts, Y. Engelbrecht, P.S. Steyn, W.H. Holzapfel, W.H. Vanzyl, Biological degradation of aflatoxin B1 by Rhodococcus erythropolis cultures. Int. J. Food Microbiol. 109, 2006, 121-126.

[37]. D. Hormisch, I. Brost, G.W. Kohring, F. Giffhorn, R.M. Kroppensted, E. Stackebrandt, P. Farber, W.H. Holzapfel, Mycobacterium fluoranthenivorans sp. nov., a fluoranthene and aflatoxin B1 degrading bacterium from contaminated soil of a former coal gas plant. J. Appl. Microbiol. 27, 2004, 653-660.

[38]. S.G. Jonathan, I.O. Fasidi, Effect of carbon, nitrogen and mineral sources on growth of Psathyerella atroumbonata (Pegler), a Nigerian edible mushroom. Food Chem. 72, 2001, 479-483.

[39]. X. Sun, X. He, Y. Li, D. Xu, H. Qian, Biological detoxification of zearalenone by Aspergillus niger strain FS10. Food Chem. Toxicol., 72, 2014, 76-82.

[40]. K. Brzonkalik, T. Herrling, C. Syldatk, A. Neumann, The influence of different nitrogen and carbon sources on mycotoxin production in Alternaria alternate. Int. J. Food Microbiol. 147, 2011, 120-126.

[41]. A. Nancib, N. Nancib, D. Meziane-Cherif, A. Boubendir, M. Fick, J. Boudrant, Joint effect of nitrogen sources and B vitamin supplementation of date juice on lactic acid production by Lactobacillus casei subsp. rhamnosus. Bioresour. Technol. 96, 2005, 6367.

[42]. G. Kohut,; A.L. Ádám, B. Fazekas, L. Hornok, N-starvation stress induced FUM gene expression and fumonisin production is mediated via the HOG-type MAPK pathway in Fusarium proliferatum. Int. J. Food microbiol. 130, $2009,65-69$.

[43]. C.H. Shu, M.Y. Lung, Effect of $\mathrm{pH}$ on the production and molecular weight distribution of exopolysaccharide by Antrodia camphorate in batch cultures. Process Biochem. 39, 2004, 931-937.

[44]. Y. Wang, B. McNeil, pH effects on exopolysaccharide and oxalic acid production in cultures of Sclerotium glucanicum. Enzym. Microb. Technol. 17, 1995, 124-130.

[45]. A. Sharma, V. Vivekan, R.P. Singh, Solid-state fermentation for gluconic acid production from sugarcane molasses by Aspergillus niger ARNU-4 employing tea waste as the novel solid support. Bioresour. Technol. 99, 2008, 3444-3450.

[46]. D.H. D'Souza, R.E. Brackett, The role of trace metal ions in aflatoxin B1 degradation by Flavobacterium aurantiacum. J. Food Prot. 61, 1998, 1666-1669.

[47]. D.H. D'Souza, R.E. Brackett, The influence of divalent cations and chelators on aflatoxin B1 degradation by Flavobacterium aurantiacum. J. Food Prot. 63, 2000, 102-105

[48]. O. Molnar, G. Schatzmayr, E. Fuchs, H. Prillinger, Trichosporon mycotoxinivorans sp. nov., A new yeast species useful in biological detoxification of various mycotoxins. System. Appl. Microbiol. 27, 2004, 661-671.

[49]. J. Varga, Z. Peteri, K. Tabori, J. Teren, C. Vagvolgyi, Degradation of ochratoxin A and other mycotoxins by Rhizopus isolates. Int. J. Food Microbiol. 99, 2005, 321-328.

[50]. R. Kriszt, C. Krifaton, S. Szoboszlay, M. Cserhati, B. Kriszt, J. Kukolya, A. Czeh, S. Feher-Toth, L. Torok, Z., Szoke, A new zearalenone biodegradation strategy using non-pathogenic Rhodococcus pyridinivorans K408 strain. PloS One 7, 2012 , e43608.

[51]. H. El-Nezami, N. Polychronaki, S. Salminen, H. Mykkanen, Binding rather than metabolism may explain the interaction of two food-grade Lactobacillus strains with zearalenone and its derivative a0-zearalenol. Appl. Environ. Microbiol. 68, $2002,3545-3549$.

[52]. S.E. Tinyiro, C. Wokadala, D. Xu, W. Yao, Adsorption and degradation of zearalenone by Bacillus strains. Folia Microbiol. 56, 2011, 321-327.

[53]. C. Joannis-Cassan, M. Tozlovanu, K. Hadejba-Medjdoub, N. Ballet, A. Pfohl-Leszkowicz, Binding of zearalenone, aflatoxin B1 and ochratoxin A by yeast based products: A rapid method for quantification of adsorption performance. J. Food Prot. 74, 2011, $1175-1185$.

[54]. A. Yiannikouris, J. Francois, L. Poughon, C.G. Dussap, G. Bertin, G. Jeminet, J.P. Jouany, Adsorption of zearalenone by $\beta$-Dglucans in the Saccharomyces cerevisiae cell wall. J. Food Prot. 67, 2004, 1195-1200.

[55]. J.P. Jouany, A. Yiannikouris, G. Bertin, The chemical bonds between mycotoxins and cell wall components of Saccharomyces cerevisiae have been identified. Archiva Zootechnica, 8, 2005, 26-50.

[56]. A. Yiannikouris, G. Andre, L. Poughon, J. Francois, C.G. Dussap, G. Jeminet, G. Bertin, J.P. Jouany, Chemical and conformational study of the interactions involved in mycotoxin complexation with $\beta$-D-glucans. Biomacromolecules, 7, $2006,1147-1155$.

[57]. E. Kiarie, S. Bhandari, M. Scott, D.O. Karuse, C.M. Nyachoti, Growth performance and gastrointestinal microbial ecology responses of piglets receiving Saccharomyces cerevisiae fermentation products after an oral challenge with Escherichia coli (K88). J. Anim. Sci. 89, 2011, 1062-1078. 